\title{
Conditioned Nicotine Withdrawal Profoundly Decreases the Activity of Brain Reward Systems
}

\author{
Paul J. Kenny and Athina Markou \\ Department of Neuropharmacology, The Scripps Research Institute, La Jolla, California 92037
}

Withdrawal from nicotine decreases the activity of brain reward systems, measured in rats by elevations of intracranial self-stimulation (ICSS) thresholds. This reward deficit is hypothesized to contribute to the persistence of the tobacco habit in tobacco smokers. Accumulating evidence suggests that aspects of drug withdrawal may become conditioned to previously neutral environmental stimuli via pavlovian conditioning processes. Here we investigated whether hedonically neutral stimuli repeatedly paired with nicotine withdrawal gained "affective valence" such that withdrawal-associated conditioned stimuli alone decreased brain reward function. Nicotinedependent rats were presented with a light/tone conditioned stimulus and injected with the nicotinic receptor antagonist dihydro- $\beta$ erythroidine ( $\mathrm{DH} \beta \mathrm{E} ; 3 \mathrm{mg} / \mathrm{kg}$ ) for $4 \mathrm{~d}$ consecutively; ICSS thresholds were assessed before and immediately after $\mathrm{DH} \beta \mathrm{E}$ injection. On the test day, the rats were presented with the conditioned stimulus and injected with saline; next, ICSS thresholds were assessed. During conditioning sessions, $\mathrm{DH} \beta \mathrm{E}$ elevated reward thresholds, and the magnitude by which thresholds were elevated increased during successive conditioning sessions. These data suggest that withdrawal-associated conditioned stimuli potentiated the magnitude of nicotine withdrawal as their motivational significance increased. Most importantly, on the test day, the conditioned stimulus alone elevated reward thresholds. Similarly, in a separate experiment, withdrawal-associated cues elevated reward thresholds in morphine-dependent rats. These data provide the first empirical verification that conditioned nicotine withdrawal may occur after exposure to withdrawalpaired cues. Moreover, these data demonstrate that withdrawal-paired conditioned stimuli attain negative affective valence and can decrease the activity of brain reward systems, mimicking the reward deficit observed during withdrawal from nicotine and other addictive drugs.

Key words: nicotine; morphine; conditioned withdrawal; pavlovian (classical) conditioning; addiction; craving; relapse

\section{Introduction}

Nicotine is the major psychoactive component of tobacco smoke responsible for tobacco addiction (Stolerman and Jarvis, 1995). Abstinence from nicotine after chronic exposure produces an aversive withdrawal syndrome in human smokers and laboratory rodents (Kenny and Markou, 2001). More recently, nicotine withdrawal has been shown to decrease the activity of brain reward systems, measured in rats by elevations of intracranial selfstimulation (ICSS) thresholds (Epping-Jordan et al., 1998). Avoidance and alleviation of this reward deficit are hypothesized to provide an important source of motivation that contributes to the persistence of the tobacco habit in smokers (Kenny and Markou, 2001).

Accumulating evidence suggests that aspects of withdrawal from various drugs of abuse may become conditioned to previ-

Received Nov. 23, 2004; revised May 23, 2005; accepted May 24, 2005.

This research was supported by the National Association for Research on Schizophrenia and Depression (P.J.K.), by Tobacco-Related Disease Research Program Grant 12RT-0231 from the state of California (A.M.), and by National Institute on Drug Abuse Grant DA11946 (A.M.). We thank Mike Arends for editorial assistance and Jessica Benedict for technical assistance. We are grateful to Drs. Stephanie Caille and George Koob for a critical reading of this manuscript.

This is publication 15747-NP from The Scripps Research Institute.

Correspondence should be addressed to Athina Markou, Department of Neuropharmacology, CVN-7, The Scripps Research Institute, 10550 North Torrey Pines Road, La Jolla, CA 92037. E-mail: amarkou@scripps.edu.

DOI:10.1523/JNEUROSCI.4785-04.2005

Copyright $\odot 2005$ Society for Neuroscience $\quad$ 0270-6474/05/256208-05\$15.00/0 ously neutral environmental stimuli through pavlovian conditioning processes (Wikler, 1973), and subsequent exposure to withdrawal-associated cues can produce a "conditioned withdrawal" state. Indeed, presentation of previously neutral environmental stimuli repeatedly paired with naloxone-precipitated opiate withdrawal produced withdrawal-like "somatic" symptoms in human opiate addicts, such as increased eye watering and respiration rate and decreased skin temperature (O'Brien et al., 1977). Similarly, conditioned opiate withdrawal decreased operant responding for food in nonhuman primates (Goldberg and Schuster, 1967) and rats (Baldwin and Koob, 1993) in a manner similar to that observed during naloxone-precipitated withdrawal. It is unclear, however, whether aspects of the nicotine withdrawal syndrome are similarly susceptible to pavlovian conditioning processes. Furthermore, it is unknown whether affective components of drug withdrawal, such as withdrawalinduced decreases in the activity of brain reward systems, may be conditioned to environmental stimuli.

Based on the above observations, we hypothesized that stimuli repeatedly paired with nicotine withdrawal may attain negative affective valence and thereby decrease the activity of brain reward systems, mimicking the reward deficit observed during unconditioned nicotine withdrawal. To test this hypothesis, we examined whether hedonically neutral (light/tone) stimuli repeatedly paired with nicotine withdrawal alone could elevate ICSS thresh- 
olds in nicotine-dependent rats. To examine whether conditioned withdrawal from other classes of drugs of abuse also could decrease brain reward function, we examined the effects of conditioned morphine withdrawal on reward thresholds. Such cueinduced reward deficits would be expected to act as a powerful source of motivation, possibly contributing to the persistence of the tobacco habit in smokers.

\section{Materials and Methods}

\section{Subjects}

Subjects were 46 male Wistar rats weighing 300-320 g at the start of the experiment. Rats were obtained from Charles River Laboratories (Raleigh, NC) and housed in groups of two or three per cage, with food and water available ad libitum. Animals were maintained in a temperaturecontrolled vivarium under a $12 \mathrm{~h}$ light/dark cycle (lights off at 8:00 A.M.). Animals were tested during the dark portion of the light/dark cycle. All animals were treated in accordance with the guidelines of the National Institutes of Health regarding the principles of animal care. Animal facilities and experimental protocols were in accordance with the Association for the Assessment and Accreditation of Laboratory Animal Care.

\section{Drugs}

(-)-Nicotine hydrogen tartrate salt ([-]-1-methyl-2-[3-pyridyl] pyrrolidine) and the high-affinity nicotinic acetylcholine receptor (nAChR) antagonist dihydro- $\beta$-erythroidine (DH $\beta \mathrm{E}$ ) were purchased from Sigma (St. Louis, MO). $\mathrm{DH} \beta \mathrm{E}$ salt was dissolved in sterile $0.9 \%$ saline and administered by subcutaneous injection in a volume of $1 \mathrm{ml} / \mathrm{kg}$ body weight.

\section{Apparatus}

Intracranial self-stimulation training and testing took place in 16 Plexiglas operant chambers $(25 \times 31 \times 24 \mathrm{~cm}$; MED Associates, St. Albans, VT). The floors of the operant chambers were constructed of parallel aluminum rods spaced $1.25 \mathrm{~cm}$ apart. One wall contained a metal wheel manipulandum that required $0.2 \mathrm{~N}$ force to rotate it one-quarter turn. The wheel ( $5 \mathrm{~cm}$ in width) extended from the wall $\sim 3 \mathrm{~cm}$. Each testing chamber was enclosed within a light- and sound-attenuated chamber $(62 \times 63 \times 43 \mathrm{~cm})$. Intracranial stimulation was delivered by constant current stimulators (Stimtech model 1200; San Diego Instruments, San Diego, CA). Subjects were connected to the stimulation circuit via flexible bipolar leads (Plastics One, Roanoke, VA) attached to gold contact swivel commutators (model SL2C; Plastics One) mounted above the chamber. The stimulation parameters, data collection, and all test session functions were controlled by a microcomputer.

\section{Surgery}

Placement of electrodes. Rats were anesthetized by inhalation of 1-3\% isoflurane in oxygen and positioned in a stereotaxic frame (David Kopf Instruments, Tujunga, CA). The incisor bar was adjusted to $5 \mathrm{~mm}$ above the interaural line, and the skull was exposed. Stainless-steel bipolar electrodes (11 $\mathrm{mm}$ in length) were implanted into the posterior lateral hypothalamus (anteroposterior, $-0.5 \mathrm{~mm}$ from bregma; mediolateral, \pm 1.7 $\mathrm{mm}$; dorsoventral, $8.3 \mathrm{~mm}$ from dura). Four indentations were made in the skull to accommodate screws that, together with the application of dental acrylic, held the electrodes in place. Animals were allowed to recover from surgery for at least $7 \mathrm{~d}$ before being trained in the ICSS procedure.

Osmotic mini-pump surgery. Rats were anesthetized by inhalation of $1-3 \%$ isoflurane in oxygen and prepared with Alzet osmotic mini-pumps (model 2ML2; Alza, Palo Alto, CA; delivering solution for a maximum of $14 \mathrm{~d}$ ) placed subcutaneously (back of the animal parallel to the spine). Pumps were filled with either $0.9 \%(\mathrm{w} / \mathrm{v})$ sterile saline or nicotine salt solution. The concentration of the nicotine salt solution was adjusted according to animal's body weight, resulting in a delivery of 9 $\mathrm{mg} \cdot \mathrm{kg}^{-1} \cdot \mathrm{d}^{-1}(3.16 \mathrm{mg} / \mathrm{kg}$, free base $)$. This dose of nicotine maintains stable plasma levels $(\sim 44 \mathrm{ng} / \mathrm{ml})$ comparable with those obtained in human smokers consuming $\sim 30$ cigarettes/d (Benowitz, 1988). After mini-pump implantation, the surgical wound was closed with $9 \mathrm{~mm}$ stainless-steel wound clips (Becton Dickinson Primary Care Diagnostics, Sparks, MD) and treated with topical antibiotic (bacitracin) ointment.

\section{Intracranial self-stimulation threshold procedure}

Animals were trained to respond according to a modification of the discrete trial current threshold procedure (Kornetsky and Esposito, 1979), which has been described in detail previously (Markou and Koob, 1992). Briefly, a trial was initiated by the delivery of a noncontingent electrical stimulus. This electrical reinforcer had a train duration of 500 $\mathrm{ms}$ and consisted of $0.1 \mathrm{~ms}$ rectangular cathodal pulses that were delivered at a frequency of 50-100 Hz. The current intensity that was delivered was adjusted for each animal and ranged from 50 to $200 \mu \mathrm{A}$. A one-quarter wheel turn within $7.5 \mathrm{~s}$ of the delivery of the noncontingent electrical stimulation resulted in the delivery of an electrical stimulus identical in all parameters to the noncontingent stimulus that initiated the trial. After a variable intertrial interval (7.5-12.5 s; average of $10 \mathrm{~s})$, another trial was initiated with the delivery of a noncontingent electrical stimulus. Failure to respond to the noncontingent stimulus within $7.5 \mathrm{~s}$ resulted in the onset of the intertrial interval. Responding during the intertrial interval delayed the onset of the next trial by $12.5 \mathrm{~s}$. Current levels were varied in alternating descending and ascending series. A set of three trials was presented for each current intensity. Current intensities were altered in $5 \mu \mathrm{A}$ steps. In each testing session, four alternating descending-ascending series were presented. The threshold for each series was defined as the midpoint between two consecutive current intensities that yielded positive scores (animals responded for at least two of the three trials) and two consecutive current intensities that yielded negative scores (animals did not respond for two or more of the three trials). The overall threshold of the session was defined as the mean of the thresholds for the four individual series. Each testing session was $\sim 30 \mathrm{~min}$ in duration. After establishment of stable ICSS thresholds, the rats were tested in the ICSS procedure once daily, except during the four consecutive conditioning sessions and on the test day, at which time the rats were tested before and immediately after their daily $\mathrm{DH} \beta \mathrm{E}$ or saline injection.

\section{Experimental design}

Rats $(n=31)$ were trained in the ICSS procedure for $\sim 14 \mathrm{~d}$ until stable ICSS thresholds were established (defined as $\leq 10 \%$ variation in thresholds for $3 \mathrm{~d}$ consecutively). Rats then were prepared with osmotic minipumps dispensing saline $(n=9)$ or nicotine hydrogen tartrate salt $(9$ $\mathrm{mg} \cdot \mathrm{kg}^{-1} \cdot \mathrm{d}^{-} ; \sim 3.16 \mathrm{mg} \cdot \mathrm{kg}^{-1} \cdot \mathrm{d}^{-1}$ nicotine base; $\left.n=22\right)$. All rats continued to be tested in the ICSS paradigm once daily after mini-pump implantation for $7 \mathrm{~d}$, with each session lasting $\sim 30 \mathrm{~min}$ in duration. Thus the animals were exposed to the ICSS chambers for at least $21 \mathrm{~d}$ before the initiation of the conditioning experiments. Furthermore, it is unlikely that the ICSS chambers serve as contextual stimuli because of latent inhibition processes. Day 7 after pump implantation was designated the preconditioning day (Pre). On day 8 after pump implantation, all rats once again had reward thresholds assessed. After this preinjection test, nicotine-dependent paired rats $(n=8)$ were injected with $\mathrm{DH} \beta \mathrm{E}(3$ $\mathrm{mg} / \mathrm{kg}$ ) and immediately after injection were placed into the ICSS chambers. Paired rats were exposed to a compound stimulus consisting of a light ( 100 pulses/min) and tone ( $\sim 60 \mathrm{~dB}$ constant tone) throughout the 30 min postinjection ICSS session. This cue/injection conditioning procedure was repeated for $4 \mathrm{~d}$ consecutively.

The control groups for the above experiment were as follows. Withdrawal rats $(n=7)$ were similarly nicotine dependent but were injected with $\mathrm{DH} \beta \mathrm{E}(3 \mathrm{mg} / \mathrm{kg})$ in the absence of the conditioned stimuli on each of the four conditioning sessions. Unpaired rats $(n=7)$ were also nicotine dependent but were injected with saline instead of $\mathrm{DH} \beta \mathrm{E}$ and were presented with the conditioned cues during the conditioning sessions. In addition, the unpaired rats also received $\mathrm{DH} \beta \mathrm{E}(3 \mathrm{mg} / \mathrm{kg})$ injections in their home cages $2-3 \mathrm{~h}$ after each of the four conditioning sessions but without exposure to the cues to ensure that their $\mathrm{DH} \beta \mathrm{E}$-precipitated withdrawal experience matched that of the paired group while avoiding the $\mathrm{DH} \beta \mathrm{E} /$ cue pairing. Finally, the control rats $(n=9)$ had mini-pumps dispensing saline and so were not nicotine dependent. These control rats were injected with $\mathrm{DH} \beta \mathrm{E}(3 \mathrm{mg} / \mathrm{kg})$ in the presence of the conditioned stimuli on each of the four conditioning sessions. Thus there were four 
groups in total (paired, withdrawal, unpaired, and control rats). On the test day, which occurred on the day immediately after the fourth and final conditioning day, the paired, unpaired, and control rats were injected with saline and presented with the light/tone stimulus during the postinjection ICSS session. Withdrawal rats were injected with saline in the absence of conditioned stimuli, and the postinjection ICSS session was initiated.

In a second experiment, we investigated whether cues repeatedly paired with morphine withdrawal also could alter reward thresholds in rats. Briefly, ICSS-trained rats $(n=15)$ were prepared with two subcutaneous $75 \mathrm{mg}$ morphine pellets. At $7 \mathrm{~d}$ after pellet implantation, during which time reward thresholds continued to be assessed each day, the morphinedependent paired rats $(n=8)$ underwent a conditioning and testing regimen similar to that described above for the nicotine-dependent paired rats, with the exception that morphinedependent paired rats were injected with the opiate receptor antagonist naloxone $(0.03 \mathrm{mg} /$ $\mathrm{kg}$ ) during conditioning sessions instead of $\mathrm{DH} \beta \mathrm{E}$. This dose of naloxone was shown previously to precipitate elevations of reward thresholds in morphine-dependent rats without affecting thresholds in morphine-naive control rats (Schulteis et al., 1994). The morphine-dependent unpaired rats $(n=7)$ underwent a conditioning and testing regimen similar to that described above for the nicotinedependent unpaired rats, with the exception that morphine-dependent rats were injected with naloxone $(0.03 \mathrm{mg} / \mathrm{kg})$ instead of $\mathrm{DH} \beta \mathrm{E}$ in their home cages $2-3 \mathrm{~h}$ after each conditioning session.

\section{Statistical analyses}

Mean absolute thresholds \pm SEM are presented for each experiment in Results. For all rats, the percentage of change from baseline reward threshold was calculated by expressing the threshold scores obtained on the preconditioning day, during the $4 \mathrm{~d}$ of cue/injection pairings, and on the test day as a percentage of the baseline thresholds minus 100. Baseline thresholds were the mean of the thresholds obtained on the three ICSS sessions immediately before the preconditioning day. For paired, withdrawal, unpaired, or control rats, the percentages of change from baseline threshold scores obtained on the preconditioning day and obtained on the $4 \mathrm{~d}$ of cue/injection pairings were compared by using one-factor repeated-measures ANOVA. After statistically significant effects in the ANOVAs, the post hoc comparisons among means were conducted with Fisher's least significant differences test. Similarly, the percentages of change from baseline threshold scores obtained on the preconditioning day and on the test day were compared by using paired Student's $t$ tests.

\section{Results}

Conditioned nicotine withdrawal elevated reward thresholds Mean \pm SEM absolute baseline reward thresholds for paired, withdrawal, unpaired, and control rats were 93.5 \pm 9.6, $114.5 \pm$ 20.0, $115.2 \pm 7.3$, and $99.1 \pm 16.5 \mu \mathrm{A}$, respectively. One-way ANOVA demonstrated no statistically significant difference in absolute baseline thresholds among these groups of rats before any treatments. Daily preinjection reward thresholds remained stable and unaltered in all four groups of rats over the $4 \mathrm{~d}$ of conditioning and on the test day (data not shown). During the $4 \mathrm{~d}$ of cue/injection pairings, the $\mathrm{DH} \beta \mathrm{E}$ administration and cue presentation elevated postinjection thresholds, which is characteris-
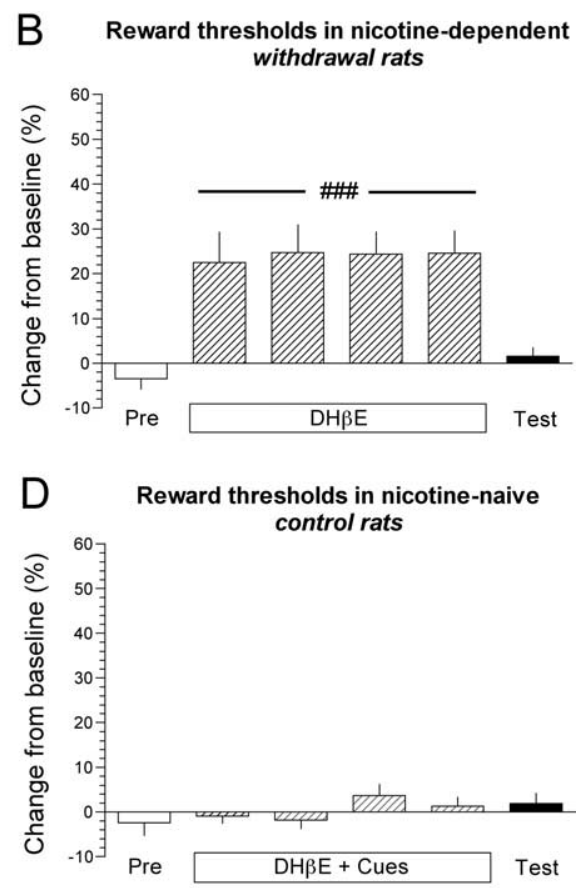

Figure 1. Conditioned nicotine withdrawal decreased the activity of brain reward systems. $\boldsymbol{A}$, Percentage of change from (he absence of cues. \#\#\# $p<0.001$ main effect of $\mathrm{DH} \beta \mathrm{E}$ on reward thresholds (one-way repeated-measures ANOVA). C, Reward thresholds in nicotine-dependent unpaired rats injected with saline instead of holds in nicotine-dependen unpaired rats

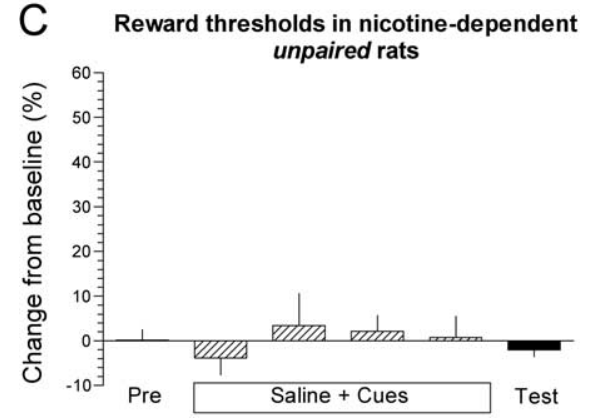
ds in nicotine-dependent paired rats
nolds in nicotin

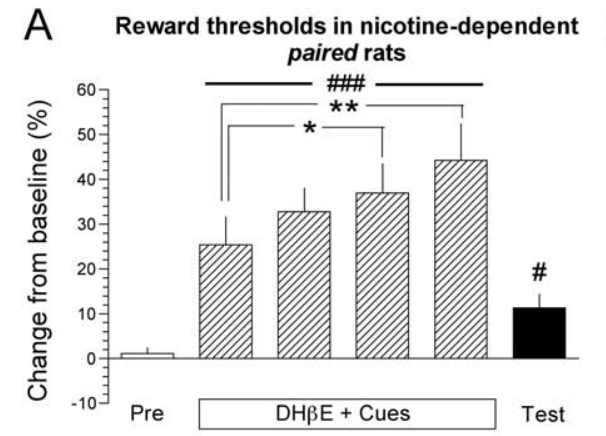

,

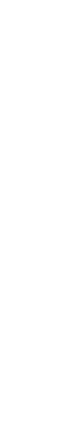



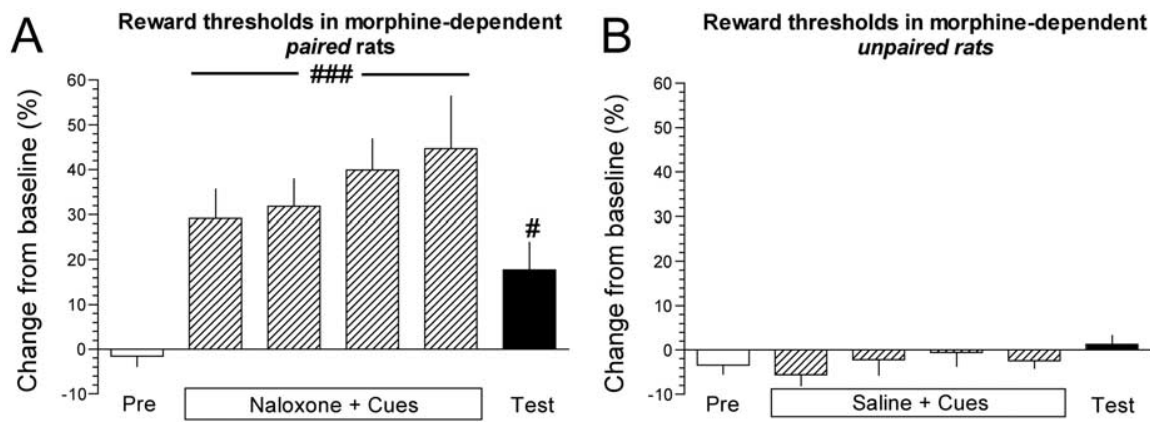

Figure 2. Conditioned morphine withdrawal decreased the activity of brain reward systems. $\boldsymbol{A}$, Percentage of change from baseline ICSS thresholds \pm SEM in morphine-dependent paired rats on the preconditioning day (Pre), during the cue/injection pairings (Naloxone + Cues), and on the test day (Test) when rats were presented with the cues and injected with saline. ${ }^{\# \#} p<$ 0.001 , main effect of naloxone/cues on reward thresholds in conditioned rats (one-way repeated-measures ANOVA); ${ }^{*} p<0.05$, thresholds on the test day compared with thresholds on the preconditioning day (paired $t$ test). $\boldsymbol{B}$, Percentage of change from baseline ICSS thresholds \pm SEM in morphine-dependent unpaired rats injected with saline instead of naloxone during conditioning sessions.

tion thresholds during the cue/injection pairings remained stable and unaltered in morphine-dependent paired and unpaired rats during the four successive conditioning sessions and on the test day (data not shown). During conditioning sessions, naloxone administration and cue presentation elevated postinjection thresholds, characteristic of opiate withdrawal (Schulteis et al., $1994)$, in the morphine-dependent paired rats $\left(F_{(4,28)}=8.6 ; p<\right.$ 0.001 ) (Fig. 2A). Although not statistically significant, the magnitude by which naloxone elevated reward thresholds demonstrated a trend toward an increase across days (Fig. 2A). Saline administration and cue presentation had no effect on postinjection reward thresholds in the morphine-dependent unpaired rats. Paired $t$ test demonstrated that postinjection thresholds on the test day were elevated significantly compared with thresholds on the preconditioning day in the paired (Fig. $2 A)(p<0.05)$, but not in the unpaired, rats (Fig. $2 B$ ).

\section{Discussion}

Addiction to tobacco smoking depends not only on the positive reinforcing and hedonic actions of nicotine but also on escape from the aversive consequences of nicotine withdrawal (Doherty et al., 1995; Kenny and Markou, 2001). The present study demonstrates that conditioned cues repeatedly paired with nicotine withdrawal significantly decreased the activity of brain reward systems, measured by elevations of ICSS thresholds in rats, mimicking the reward deficit observed during unconditioned nicotine withdrawal. Similarly, conditioned morphine withdrawal also elevated reward thresholds, suggesting that cues associated with withdrawal from different classes of addictive drugs share the ability to decrease brain reward function. The cue-induced reward deficits reported here were similar to those observed during unconditioned withdrawal from most major drugs of abuse, including nicotine (Epping-Jordan et al., 1998), morphine (Schulteis et al., 1994), alcohol (Schulteis et al., 1995), and cocaine (Markou and Koob, 1991). Avoidance and alleviation of such withdrawal-induced reward deficits are considered a major source of motivation contributing to continued drug use and relapse during abstinence (Ahmed et al., 2002). Thus environmental stimuli repeatedly paired with drug withdrawal potentially may drive drug-seeking and drug-taking behavior, in part by precipitating a withdrawal-like reward deficit and thereby setting the occasion for drug-dependent individuals to selfmedicate such reward deficits by means of additional drug con- sumption. Drug-paired cues are known to produce drug-like responses and also have been proposed to be a major source of motivation contributing to drug use and reinstatement (Stewart et al., 1984). Thus it will be important to decipher the interplay between conditioned positive and conditioned negative effects in drug-taking and craving (Hutcheson et al., 2001).

The magnitude of the unconditioned effect (i.e., antagonist-precipitated withdrawal) was similar in nicotine-dependent and morphine-dependent rats. However, the reward deficit observed during conditioned morphine withdrawal appeared greater than that during conditioned nicotine withdrawal. One possible explanation for this observation is that nAChRs may be involved in associative learning and that inhibition of these receptors during conditioning sessions attenuated the conditioning process. Whatever the explanation, it is important to note that the magnitude by which conditioned nicotine or conditioned morphine withdrawal elevated reward thresholds was diminished greatly when compared with the unconditioned effect in these rats. Nevertheless, the relatively small magnitude of the conditioned effects compared with the unconditioned effects is consistent with the assumptions of pavlovian conditioning theory (Pavlov, 1927) and also with previous experimental observations (Kenny et al., 2003).

$\mathrm{DH} \beta \mathrm{E}$ was chosen for the present studies because, unlike the noncompetitive $\mathrm{nAChR}$ antagonist mecamylamine, we have shown previously that $\mathrm{DH} \beta \mathrm{E}$ precipitates withdrawal-associated reward deficits in nicotine-dependent rats without precipitating somatic aspects of nicotine withdrawal (Epping-Jordan et al., 1998). This approach allowed us to examine whether affective aspects of nicotine withdrawal were susceptible to pavlovian conditioning while avoiding potentially confounding influences of somatic aspects of withdrawal on conditioning processes. It is interesting to note that, during the conditioning sessions, the magnitude by which $\mathrm{DH} \beta \mathrm{E}$ elevated reward thresholds in nicotine-dependent paired rats progressively increased over the 4 conditioning days. Because reward thresholds assessed before each conditioning session remained stable and unaltered, this progressive increase in withdrawal magnitude cannot be attributed to a carryover of the threshold-elevating effects of $\mathrm{DH} \beta \mathrm{E}$ from the previous day such that each successive withdrawal was precipitated before the previous withdrawal had abated. Furthermore, we also show that $\mathrm{DH} \beta \mathrm{E}$ administered to nicotinedependent withdrawal rats under a similar injection schedule just before the ICSS session but without the presentation of conditioned stimuli resulted in stable threshold elevations ( $\sim 25 \%$ elevation of thresholds each day; no progressive increase in the unconditioned withdrawal response). Thus the progressive increase in withdrawal magnitude in paired rats reported here likely reflects a potentiation of withdrawal induced by conditioned stimuli as their motivational significance increases. Consistent with this hypothesis, withdrawal-induced elevations of reward thresholds in morphine-dependent rats also tended to increase across days when paired with conditioned stimuli, although this effect did not reach statistical significance. However, a recent study demonstrated that the magnitude by which naloxone suppressed operant responding for food reinforcement in opiate- 
dependent rats was increased significantly when naloxone was administered in a specific environment (Schulteis et al., 2004), suggesting that contextual cues may potentiate withdrawal. Overall, these data suggest the possibility that, as discrete and contextual stimuli become paired repeatedly with nicotine withdrawal in human smokers, the magnitude of withdrawal-induced reward deficit may become progressively greater, potentially contributing more and more to the persistence of the tobacco habit. It is important to note, however, that nicotine-dependent withdrawal rats (Fig. $1 B$ ) that did not show a potentiation of withdrawal magnitude underwent a $\mathrm{DH} \beta \mathrm{E}$ injection procedure and exposure to the ICSS chambers similar to the paired rats. It may have been expected that environmental cues other than the experimenter-presented conditioned stimuli, which were not presented to withdrawal rats, may have become associated with $\mathrm{DH} \beta \mathrm{E}$ administration and thereby potentiated nicotine withdrawal across conditioning days. One explanation for the absence of such an effect is that withdrawal rats were exposed to the ICSS chambers for at least $21 \mathrm{~d}$ before the first $\mathrm{DH} \beta \mathrm{E}$ injection. Such extensive preexposure to the testing environment in withdrawal rats may have induced a latent inhibition, defined as the process by which preexposure to a stimulus without consequence decreases the capacity to establish subsequent conditioned associations with that stimulus.

The present data are an extension of the groundbreaking work of Wikler (1948), who first proposed that cues repeatedly paired with drug withdrawal may elicit a conditioned withdrawal state. Indeed, O’Brien et al. (1977) demonstrated that withdrawalassociated cues produced an aversive conditioned withdrawal state in human opiate addicts, consistent with anecdotal reports that addicts experienced withdrawal symptoms when they encountered drug-related environmental stimuli (O'Brien et al., 1975). Importantly, previous investigations of conditioned drug withdrawal in humans or laboratory animals measured cueinduced physical aspects of withdrawal (O'Brien et al., 1977) or a cue-induced suppression of responding for food reinforcement behavior (Baldwin and Koob, 1993). However, it has been hypothesized that avoidance of the affective components of withdrawal may play a more important role in the maintenance of dependence to drugs of abuse, including nicotine, than the somatic aspects of withdrawal (Watkins et al., 2000; Kenny and Markou, 2001). Thus the present data crucially demonstrate that withdrawal-associated conditioned stimuli gain affective valence and thereby precipitate affective components of drug withdrawal, such as the reward deficit usually observed during unconditioned withdrawal from nicotine and other major drugs of abuse. The present studies also highlight an important role for learning and memory pathways in regulating the activity of reward systems in the brain, the cross-communication between which likely plays an important role in the persistence of drug-taking behavior (Everitt et al., 2001). Nevertheless, it is important to note that the effects of withdrawal-associated cues were not examined in rats after the removal of nicotine-delivering mini-pumps or morphine pellets. Thus future studies are required to determine whether conditioned deficits in brain reward function occur in postdependent rats.

In conclusion, the present data demonstrate that environmental stimuli repeatedly paired with nicotine withdrawal gain negative affective valence and can elicit a deficit in brain reward function that mimics the reward deficit observed during unconditioned drug withdrawal. Because withdrawal-associated defi- cits in brain reward function are hypothesized to provide an important source of motivation that maintains and relapses drugtaking behavior, the present data suggest that cue-induced decreases in brain reward function may generate an important source of craving contributing to the persistence of the tobacco habit in smokers.

\section{References}

Ahmed SH, Kenny PJ, Koob GF, Markou A (2002) Neurobiological evidence for hedonic allostasis associated with escalating cocaine use. Nat Neurosci 5:625-626.

Baldwin HA, Koob GF (1993) Rapid induction of conditioned opiate withdrawal in the rat. Neuropsychopharmacology 8:15-21.

Benowitz NL (1988) Pharmacological aspects of cigarette smoking and nicotine addiction. N Engl J Med 319:1318-1330.

Doherty K, Kinnunen T, Militello FS, Garvey AJ (1995) Urges to smoke during the first month of abstinence: relationship to relapse and predictors. Psychopharmacology (Berl) 119:171-178.

Epping-Jordan MP, Watkins SS, Koob GF, Markou A (1998) Dramatic decreases in brain reward function during nicotine withdrawal. Nature 393:76-79.

Everitt BJ, Dickinson A, Robbins TW (2001) The neuropsychological basis of addictive behaviour. Brain Res Brain Res Rev 36:129-138.

Goldberg SR, Schuster CR (1967) Conditioned suppression by a stimulus associated with nalorphine in morphine-dependent monkeys. J Exp Anal Behav 10:235-242.

Hutcheson DM, Everitt BJ, Robbins TW, Dickinson A (2001) The role of withdrawal in heroin addiction: enhances reward or promotes avoidance? Nat Neurosci 4:943-947.

Kenny PJ, Markou A (2001) Neurobiology of the nicotine withdrawal syndrome. Pharmacol Biochem Behav 70:531-549.

Kenny PJ, Koob GF, Markou A (2003) Conditioned facilitation of brain reward function after repeated cocaine administration. Behav Neurosci 117:1103-1107.

Kornetsky C, Esposito RU (1979) Euphorigenic drugs: effects on the reward pathways of the brain. Fed Proc 38:2473-2476.

Markou A, Koob GF (1991) Postcocaine anhedonia. An animal model of cocaine withdrawal. Neuropsychopharmacology 4:17-26.

Markou A, Koob GF (1992) Construct validity of a self-stimulation threshold paradigm: effects of reward and performance manipulations. Physiol Behav 51:111-119.

O’Brien CP, O’Brien TJ, Mintz J, Brady JP (1975) Conditioning of narcotic abstinence symptoms in human subjects. Drug Alcohol Depend 1:115-123.

O’Brien CP, Testa T, O’Brien TJ, Brady JP, Wells B (1977) Conditioned narcotic withdrawal in humans. Science 195:1000-1002.

Pavlov IP (1927) Conditioned reflexes: an investigation of the physiological activity of the cerebral cortex (Anrep GV, ed, translator). London: Oxford UP.

Schulteis G, Markou A, Gold LH, Stinus L, Koob GF (1994) Relative sensitivity to naloxone of multiple indices of opiate withdrawal: a quantitative dose-response analysis. J Pharmacol Exp Ther 271:1391-1398.

Schulteis G, Markou A, Cole M, Koob GF (1995) Decreased brain reward produced by ethanol withdrawal. Proc Natl Acad Sci USA 92:5880-5884.

Schulteis G, Morse AC, Liu J (2004) Conditioning processes contribute to severity of naloxone-precipitated withdrawal from acute opioid dependence. Psychopharmacology (Berl) 175:463-472.

Stewart J, de Wit H, Eikelboom R (1984) Role of unconditioned and conditioned drug effects in the self-administration of opiates and stimulants. Psychol Rev 91:251-268.

Stolerman IP, Jarvis MJ (1995) The scientific case that nicotine is addictive. Psychopharmacology (Berl) 117:2-20.

Watkins SS, Koob GF, Markou A (2000) Neural mechanisms underlying nicotine addiction: acute positive reinforcement and withdrawal. Nicotine Tob Res 2:19-37.

Wikler A (1948) Recent progress in research on the neurophysiological basis of morphine addiction. Am J Psychiatry 105:329-338.

Wikler A (1973) Dynamics of drug dependence. Implications of a conditioning theory for research and treatment. Arch Gen Psychiatry 28:611-616. 